\title{
Distributed Implementation of Discrete Event Control Systems based on Petri Nets
}

\author{
Ramón Piedrafita Moreno, Danilo Tardioli, José Luis Villarroel Salcedo \\ Department of Computer Science and Systems Engineering, University of Zaragoza, Spain \\ \{piedrafi,dantard,jlvilla\}@unizar.es
}

\begin{abstract}
In this paper, we propose a platform for the implementation of Distributed discrete event control systems. We propose an architecture for the Distributed implementation of Petri Nets (PN) in control applications. The architecture covers the local execution of $P N$ and the communication amongst controllers. Both synchronous and asynchronous communication paradigm is supported. A framework of classes for supporting the real time communication and real time execution or remote calls has been developed. The Real Time Wireless Multi-hop Protocol has been adopted. With this protocol and the framework of classes developed, control deadlines of the system can be guaranteed. In order to solve access to common resources, a new class of communications places, the Auction Communication Places, is proposed. To support the real time and concurrent characteristics, we used the Java real-time specification. In order to implement $P N$, we have extended the concurrent coordinators technique, developed in previous works, to distributed systems introducing the distributed coordinators. To demonstrate the practical utility and feasibility of the architecture, we have applied it to the control of a flexible manufacturing cell.
\end{abstract}

\section{INTRODUCTION}

This paper deals with the development of distributed control systems. In previous works [1] a centralized control architecture and a development environment based on Petri Nets and Java language was developed. In the present work, this control architecture is extended to be applied to distributed control systems.

The hardware of the proposed control architecture is based on several computers, connected by communications networks, and fieldbus input/output modules for machine control.

The Petri Net (PN), that specify the control, is distributed and then implemented in several computers of the control architecture. The mechanisms that support the communication between PNs allow the communication in asynchronous form by means of communication places, and in synchronous form by means of shared transitions. These high level communication paradigm, must be implemented over a real time communication network for guarantee the control temporal requirements. For this purpose, the RTWMP protocol [2] developed at the University of Zaragoza has been adopted.

From the perspective of the software implementation of $\mathrm{PN}$, we have developed a new technique called distributed coordinators that extend a previous one, the concurrent coordinators [1]. And to solve conflicts between distributed processes we propose a new technique: the Auction Communication Place.

A practical application of the proposed approach was developed at the Department of Computer Science and Systems Engineering at the University of Zaragoza, Spain: the distributed control of a flexible manufacturing cell.

This article is organized as follows: in Section II, we review the different techniques used to implement PN. Section III describes distributed implementation of PN. and in Section IV the characteristics of the RT-WMP based implementation are indicated. The application over the flexible manufacturing cell is described in Section V. In Section VI, we present conclusions and suggest future lines of research.

\section{SOFTWARE IMPlementation OF PETRI NetS}

A Petri net is a graphical, mathematical tool that can be used to model discrete event systems [3]. PN are bipartite graphs that have two types of nodes: places and transitions, which are represented by circles and bars respectively. Directional arcs connect places and transitions. Input arcs connect places with transitions, and output arcs connect transitions to places. Places contain a positive or null number of tokens. The token distribution over PN places is called marking and represents the system state. Transitions represent events. A transition firing models the occurrence of an event that changes the system state (the marking of the PN).

In the last 25 years, researchers have devoted considerable attention to the software implementation of $\mathrm{PN}$; see for example [4] [5] [6] [7] [8]. A software implementation is a program that triggers the firing of the net transitions observing the marking evolution rules, that is, it plays the token game. Depending on the criteria, a Petri net implementation can be classified as compiled or interpreted, sequential or concurrent, and centralized or decentralized. An implementation is interpreted if the net structure and the marking are codified as data structures. The data structures are used by one or more tasks called interpreters to establish the net evolution. The interpreters do not depend on the implemented net. A compiled implementation is based on the 
generation of one or more tasks whose control flow corresponds to net evolutions.

In a centralized implementation, the token player is executed by a single task, which is called the coordinator. To characterize a centralized implementation, the algorithm to determine which transitions are enabled and can fire is important. Apart from the simple exhaustive test of all of the transitions, there are various solutions for reducing the costs of the enabling test, including static or dynamic representing places [8] and transition-driven techniques [7].

In a decentralized implementation, the net is decomposed into a set of sequential structures. Each of the structures is implemented by a task and the implementation is completed using the necessary communication and synchronization mechanisms.

In [1] we have introduced centralized techniques into decentralized implementations, thereby creating a new technique called concurrent coordinators. The application can run several coordinators simultaneously by executing a sub-net for each subsystem. In this technique the net can be split following control or functional criteria, different from the behavioral ones needed for other decentralized techniques.

In this work we developed the distributed implementation of PN, extending the previous concurrent coordinators technique. We call this technique the distributed coordinators. In it, the execution of the coordinators takes place in several hosts, connected by a communication network. In each host one or more coordinators executing its Petri net. The coordinators read the inputs, executing its Petri net (the token player) and writes the outputs, i.e. making the local machine control. For the necessary information interchange between hosts, the implementation is completed using the necessary communication mechanisms.

A Middleware that allows the implementation of distributed PN has been developed. For this purpose, a set of Java Classes to implement synchronous and asynchronous communication paradigms has been designed. These classes implement the local and remote communications places, the auction communication place and the auction solver.

For implement the communication between the different coordinators will be needed a protocol that support real time traffic over Ethernet. In our case has been chosen the protocol RT-WMP, but the same implementation could have been made with other protocols real time like RT-RMI [9] or RTEP [10].

The classes to manage communication with the Real Time RT-WMP protocol have been developed. This set of classes also implements the necessary real time execution of the remote procedure calls. Also, the classes for communicating with input/output modules in industrial field buses CANopen, Interbus and Industrial Ethernet have been developed.

We have implemented the distributed implementation techniques of PN in the Java language using the Java Real Time extension [11] and following some ideas presented in
[1] [12]. The real time extension provides Java with the necessary aspects for the programming of real-time systems, e.g., pre-emptive planning based on static priorities, asynchronous transfer of control, real-time high resolution clocks, and the possibility of execution over the Java garbage collector. In our implementations, we used the Real Time Java Virtual Machine JamaicaVM v2.7 [13]. The target hardware was a personal computer with Pentium IV processor at $1.7 \mathrm{GHz}$, running Red Hat Linux 2.4.

\section{Distributed IMPLEMENTATION OF PETRI NetS}

In order to make a distributed implementation of PN, a net partition has to be made. In previous works [8] [14] [15] the authors show how to make a Petri net partition in sequential processes, applying structural and behavioral analysis. In this work the partition is done following a functional criterion. The PN are subdivided in several subnets local access to the input/output modules criteria and the machine's local control criteria.

This partition sets out a set of subnets, a set of shared places and a set of shared transitions. The subnets can be implemented in several computers. The implementation of the subnets is made in a centralized form. In each computer one or more coordinators are in charge of executing the corresponding subnets. The coordinators are threads with real-time requirements. From the perspective of the Java Real Time Specification, they are periodic RealtimeThreads with high priority that are scheduled without round-robin.

The shared places model the asynchronous communications; the shared transitions model the synchronous communication. The communication between distributed PN can be made in asynchronous form by means of shared places, and in synchronous form by means of shared transitions (see Fig. 1).

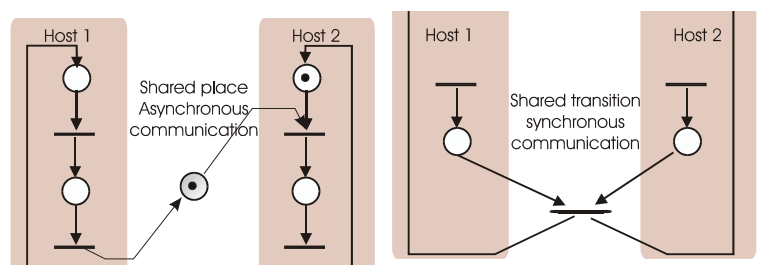

Fig. 1. Asynchronous and synchronous Communication.

\section{A. Communications Places.}

The partition in two or more subnets brings to the appearance of shared places amongst subnets. These places are known as communication places (see Fig. 2). A communication place is implemented as a protected object with synchronized methods to mark and unmark the place. Program 1 shows the communication place code.

The communications places can be shared between Petri nets of the same host, or between Petri nets of different hosts. A communication place is implemented in one of the hosts and belongs to the PN executed in that machine. 


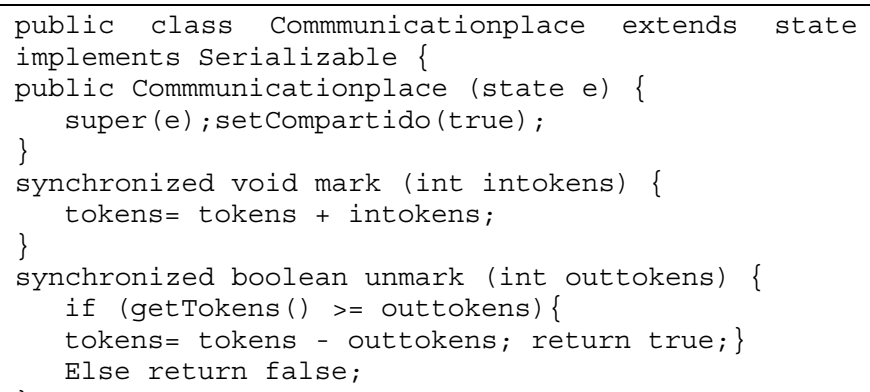

The interface of communication places is also implemented in the rest of the nodes that share it, as communication places remote interface. The calls to the methods of communication places remote interface are sent to communication places through the real time communication network.

It is possible to access this place as:

- communication place, when the access is local in the host machine. The local coordinator and its communications places are implemented in the same Java Virtual machine. The Local coordinator access to its communication places callings is methods directly.

- communication place remote interface (CPRI), when the access is from another machine of the network. The CPRI is the remote implementation of the corresponding communication place. The CPRI and the remote coordinator are implemented in a remote host in its Java Virtual Machine. The Remote coordinator access to its CPRI callings is methods directly. These callings are directed to the communication places through the real-time communication network over the protocol RT-WMP.
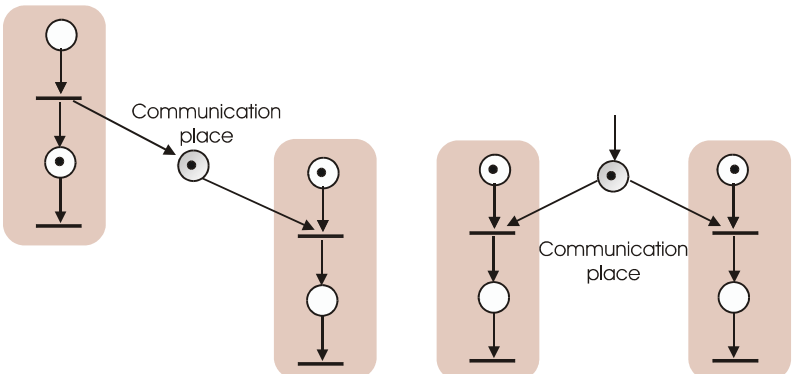

Fig. 2. Communication place: a) single destination b) multiple destination

B. Auction Communications Places.

If, in the execution of its Petri net, a coordinator needs to unmark a communication place, it is necessary to consult the number of available tokens and then unmark the communication place. This fact can cause problems since operation is not atomic. In our decentralized implementation [1], several coordinators, all of them implemented like Real Time Threads with the same priority, run simultaneously in a single computer. The execution is made in a single processor and threads are scheduled following a static priorities policy without round-robin. A coordinator can consult the number of tokens in a place and next fire the transition and unmark the place without pre-emption by another coordinator.
However, if implementation is distributed, the simultaneous execution of several coordinators takes place in several computers, and it can happen that several coordinators want to unmark a communication place simultaneously. These external conflicts can occur when a communication place has several output transitions distributed in different subnets. In this case, the conflict has been solved by means of the introduction of an auction where the transitions are fired in priority order. These shared places are implemented by means of a protected object called auction communication place.

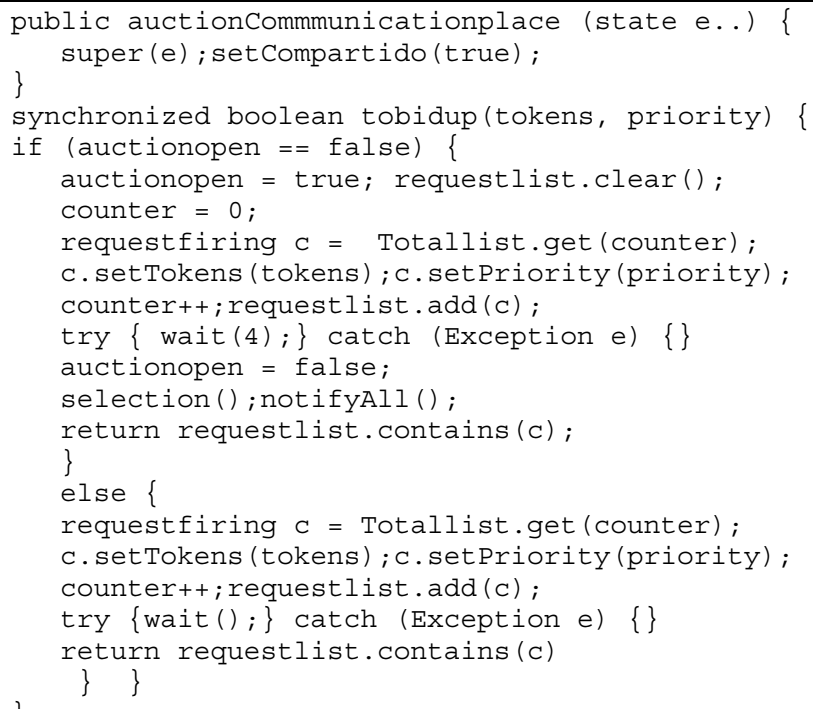

When a coordinator wants to unmark an auction communication place, it carries out a call to the method tobidup () of this one. The call is stored with its priority and the number of tokens requested. Next, if it is the first request, the caller (i.e. the first coordinator) is suspended for $4 \mathrm{~ms}$. Coordinators are implemented like Periodic Real Time Threads with $25 \mathrm{~ms}$ of period to obtain good control of the machines. The conflict must be solved within the $25 \mathrm{~ms}$ of period of the coordinators. During this $4 \mathrm{~ms}$, other local or remote coordinators can enter the auction to unmark that place. Its requests are stored and they are suspended by calling a wait() method. After $4 \mathrm{~ms}$, the first coordinator wakes up and closes the auction. Then it calls to the method selection() to assign tokens, and next with notifyall() wakes up the rest of the coordinators. If the assigned request list contains the request of the coordinator, the method tobidup () returns true. The execution of a tobidup() call consists of the execution of the test and the unmarking of the auction communication place. These two operations are made in atomic form.

In this way, the conflict is correctly solved, since all the transitions that want to unmark a place enter in the auction and the transitions are fired in priority order. With this method, remote coordinators can access the tokens. The 
maximum blocking time is $4 \mathrm{~ms}$ to avoid control problems, but also to allow the calls to tobidup() that travel through the real time communication network to be taken into account.

In case of distributed simple conflicts where the communication places have several output transitions in the same PN, the implementation of the communication place must be made in an active object, in a thread. If several transitions that belong to the same PN wish to unmark the same communication place, the first call of tobidup() blocks the execution of the coordinator, thus the rest of transitions would not enter the auction. Therefore, to solve this problem, an active object that manages these situations is needed. We call it auction solver.

The auction solver receives requests of the output transitions, reserves tokens according to the priority and informs in a list which transitions can fire. When the suspend time is expired and the place is not unmarked by the transition, the auction solver cancels the reservation of the marks. The implementation of the auction solver is made up in a Real Time Thread of higher priority than the coordinator Thread.

\section{Synchronous Communication}

For the implementation of shared transitions in Java, it would be necessary to have some primitive of synchronous communication. Java does not have instructions that allow the synchronous communication like the ADA rendez-vous. Moreover, even if it would be available, it could not be used since the rendezvous construction blocks the caller thread. Therefore the coordinator is blocked until the acceptance of the appointment and it involves the loss of the control during that time. In order to implement the shared transitions in Java it is necessary to transformation the PN according to Fig. 3. In this way, a synchronous communication using a shared transition is transformed into two asynchronous communications using communication places.
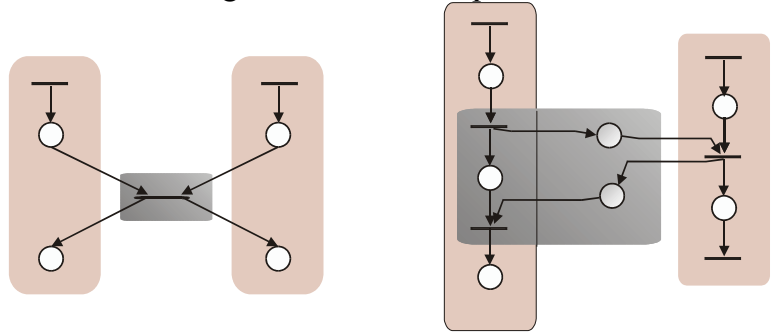

Fig. 3. Conversion of a synchronous communication in two asynchronous communications.

\section{REAL TIME COMMUNICATION}

In order to guarantee a bounded time response, real-time communication is necessary amongst the nodes of the PN, and the execution of the methods should be made with the appropriate real time priority.

The remote access to a shared place is made by means of messages in protocol RT-WMP. The Fig. 7 shows the access from the coordinators executed in the virtual machines of PC1 and PC3 to a shared place implemented in the virtual machine of the PC2. The access is made locally to the CPRI. The calls to the methods of CPRI are transformed into messages in protocol RT-WMP. These messages when arriving at the PC2 suppose the execution of methods of the communication place.

The RT-WMP is a novel protocol that supports hard realtime traffic. In this sense in RT-WMP, end-to-end messages delay has a bounded and known duration and it manages global static priorities of messages. The protocol has been though to connect a relatively small group of nodes. It is based on a token passing scheme. RT-WMP has a built-in error recovery mechanism that can recover from certain types of errors without jeopardizing the real-time behavior. The RT-WMP can run over commercial hardware without modifications. It is currently implemented on the Linux platform and uses commercial low-cost Ethernet devices. In RT-WMP each node has a transmission and a reception priority queue.

Each message is identified by a priority level and message with the same priority is stored in FIFO order. When an application needs to transmit a message to another node, it pushes it in the transmission queue. The RT-WMP process pops the message from that queue and transmits it through the network to the destination node. The latter pushes the message in the reception queue and the destination application can finally pop the message from that queue.

Even if the RT-WMP has been designed to work in wireless multi-hop networks, it can work perfectly over wired Ethernet. However the nodes belonging to the RT-WMP protocol must have a dedicated and isolated subnetwork to guarantee the real-time performances. The RT-WMP is written in $\mathrm{C}$ language. We have developed an interface Java to $\mathrm{C}$ with Java Native interface technology (JNI). A dynamic library has been created and their procedures have been adapted to be used from Java using the JNI.

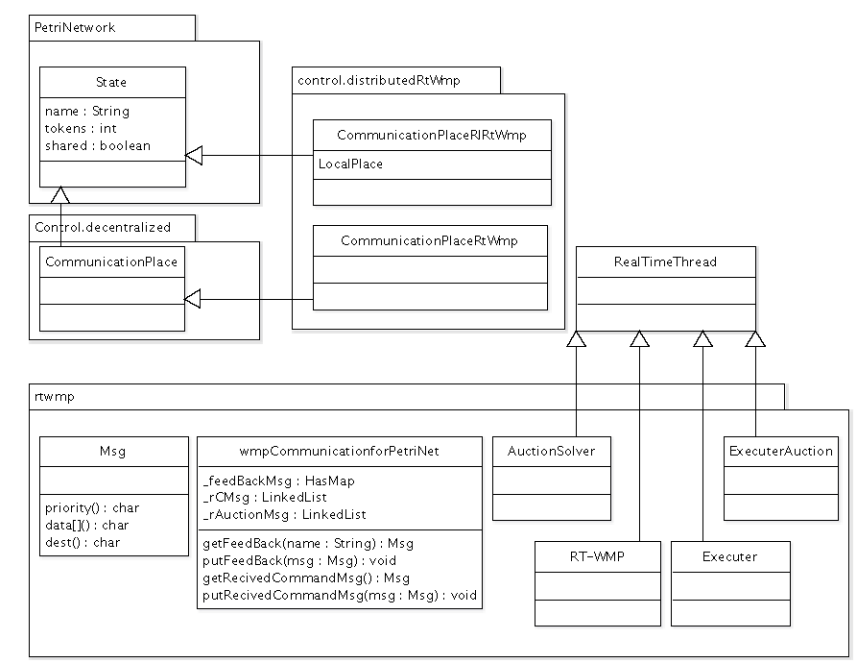

Fig. 4. RT-WMP communication Java classes. 
Fig. 4 shows the Unified Modelling Language (UML) Diagram of the classes written for RT-WMP communication. Two classes to represent the communication places have been implemented:

- CommunicationplaceRtWmp: for representation of the local instance of the object for which we want to invoke the methods.

- CommunicationplaceRIRtWmp: which is the remote interface of the Communicationplace.

The threads in charge of managing the communication and the execution of the remote calls are:

- $\quad R T-W M P$. It is a Real Time Thread of high priority; it manages the communication in the real time protocol.

- Executer. It is a Real Time Thread in charge of execution the remotes request.

- ExecuterAuction. It is similar to Executer but specialized for the tobidup() remote method because that method needs more time for its execution.

- Auction Solver. It is a Real Time Thread in charge of solving conflicts.

It is necessary that the tasks in charge of managing the communication were executed at higher priority than the coordinators. In order to obtain this:

- Access to the synchronized methods of the communication places is made by means of the priority ceiling protocol.

- The execution of the Auction Solver is made with a higher priority than the coordinators.

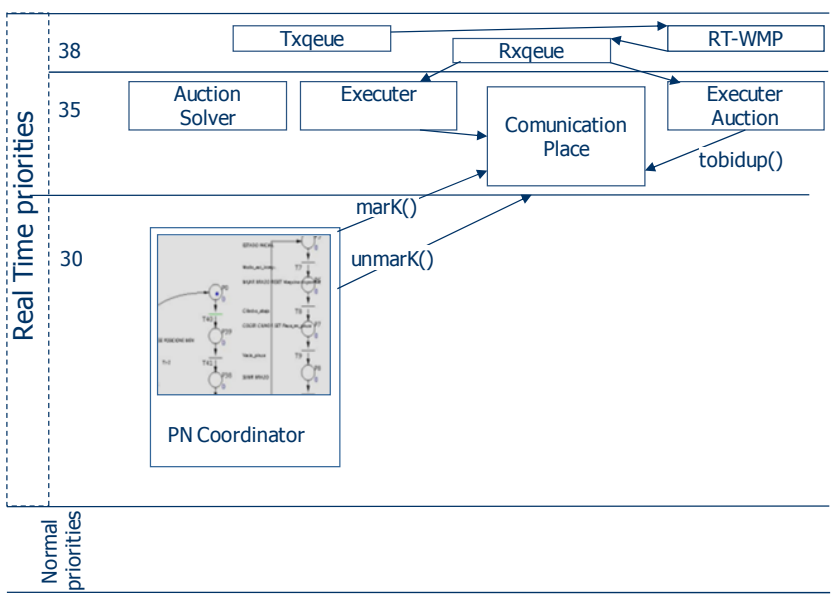

Fig. 5. Execution Priorities

- The execution of threads that deal with the communication have a priority higher than the coordinator threads.

- Threads in charge of executing the remote procedures are executed at a priority higher than the coordinators.
Thread $R T-W M P$ is executed at the maximum real time priority (in our application, 38). Threads Executer and ExecuterAuction and Auction Solver are executed with a lower priority than the $R T-W M P$ threads but greater than the Coordinators (in our application, 35). With this communication architecture a bounded response time in communication is obtained. Fig. 5 shows the Execution priorities of the threads in the proposed architecture.

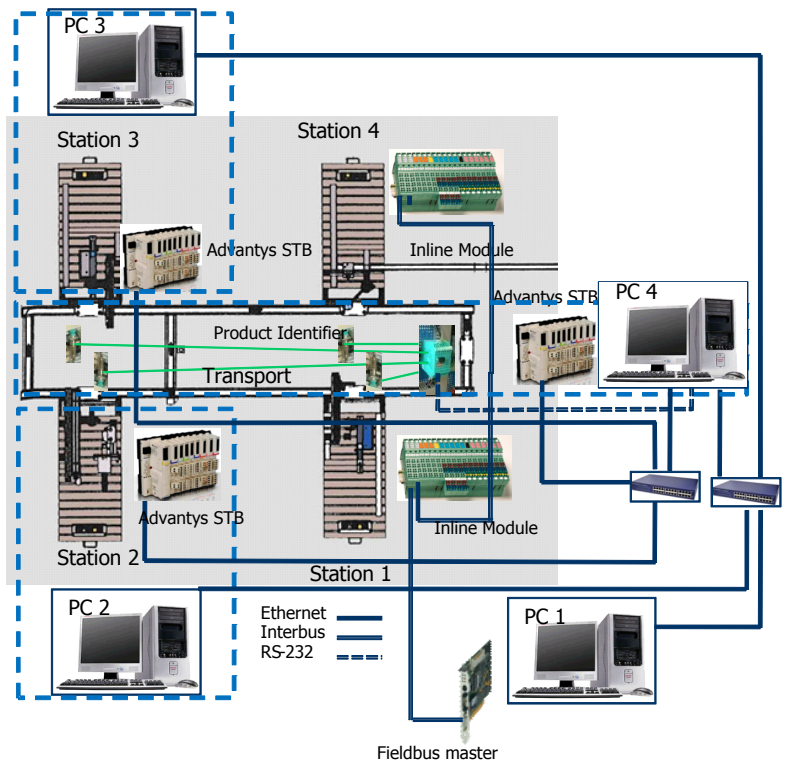

Fig. 6. Hardware Architecture

\section{Distributed Control Of The Flexible MANUFACTURING CELL}

The practical application of the technologies presented in this paper were tested using a flexible manufacturing cell installed at the Department of Computer Science and Systems Engineering at the University of Zaragoza, Spain, for research and teaching purposes. The manufacturing cell is composed of a set of stations for the production and storage of pneumatic cylinders.

The initial control system of the cell consisted of a programmable logic controller (PLC) that controlled each station. A real-time industrial net connected all of the PLCs.

We have installed a new distributed control system based on several computers, connected by communications networks, and a fieldbus (see Fig. 6). One of the computers is equipped with a fieldbus master card, where the input/output modules of the machines are connected. The rest of computers control the input/output hardware by Industrial Ethernet.

Stations 1 and 4 have Inline modules [16] with protocol Interbus, the master of the bus will be a Computer (PC 1) with the CIF50-IBM card of Hilscher [17]. In the rest of the cell, Industrial Ethernet is supported. In Stations 2, 3 and in the transport, the Advantys [18] input/output modules communicate in the protocol Modbus TCP over Ethernet. For Ethernet communication two segmented subnetworks are 
arranged. The first one is used for communication with input/output modules and the second one for real time communication amongst computers in RT-WMP protocol.

Each station of the cell has a read/write head of the pallet memory that is connected to an identifying module of products (Pepperl\&Fuchs IVI-KHD2-4HRX) [19]. The product identifier module is a resource shared by all stations. Communication with the identifier module is achieved using a serial port. Access to the module must be protected from concurrent access.

In our implementations, we used the Real Time Java Virtual Machine JamaicaVM v2.7 [13]. The target hardware was four computers with Pentium IV processor at $1.7 \mathrm{GHz}$, running Red Hat Linux 2.4.

\section{A. Software Control Architecture}

The PN that models the desired behavior of the cell has been subdivided in several subnets by following local access to the input/output modules criterion, and the local control of the machines criterion. This subdivision leads to a set of subnets and to a set of shared places that model the communications. The subnets have been implemented in several computers. Each computer is in charge of control of a part of the Cell. In each computer one or more coordinators are in charge of executing the corresponding subnet (see Fig. 7).

The execution of the coordinator takes place in a high priority thread within the Jamaica Real-Time Java Virtual Machine. PN communicate by means of shared places (i.e. the communication places). For example, the access to the product identifier is modeled by means of a shared place that is implemented as a local communication place in the PN executed by the Cell Coordinator and as a remote communication place in the rest of $\mathrm{PN}$.

\section{B. Communication}

The Fig. 8 shows the UML sequence diagram [20] of the tobidup () communication and remote execution. When in the execution of a coordinator, it is desired to unmark a communication place, it is called its method tobidup() of the CommunicationplaceRIRtWmp. This creates a message and puts it in the transmission queue of RT-WMP. This message is received by the RT-WMP of the another node. The Executer reads the message, searches the corresponding CommunicationplaceRtWmp and executes the request method. Finally sends a feedback. message to the CommunicationplaceRIRtWmp. The time necessary to unmark a communication place from a remote machine is between 10 and $12 \mathrm{~ms}$.

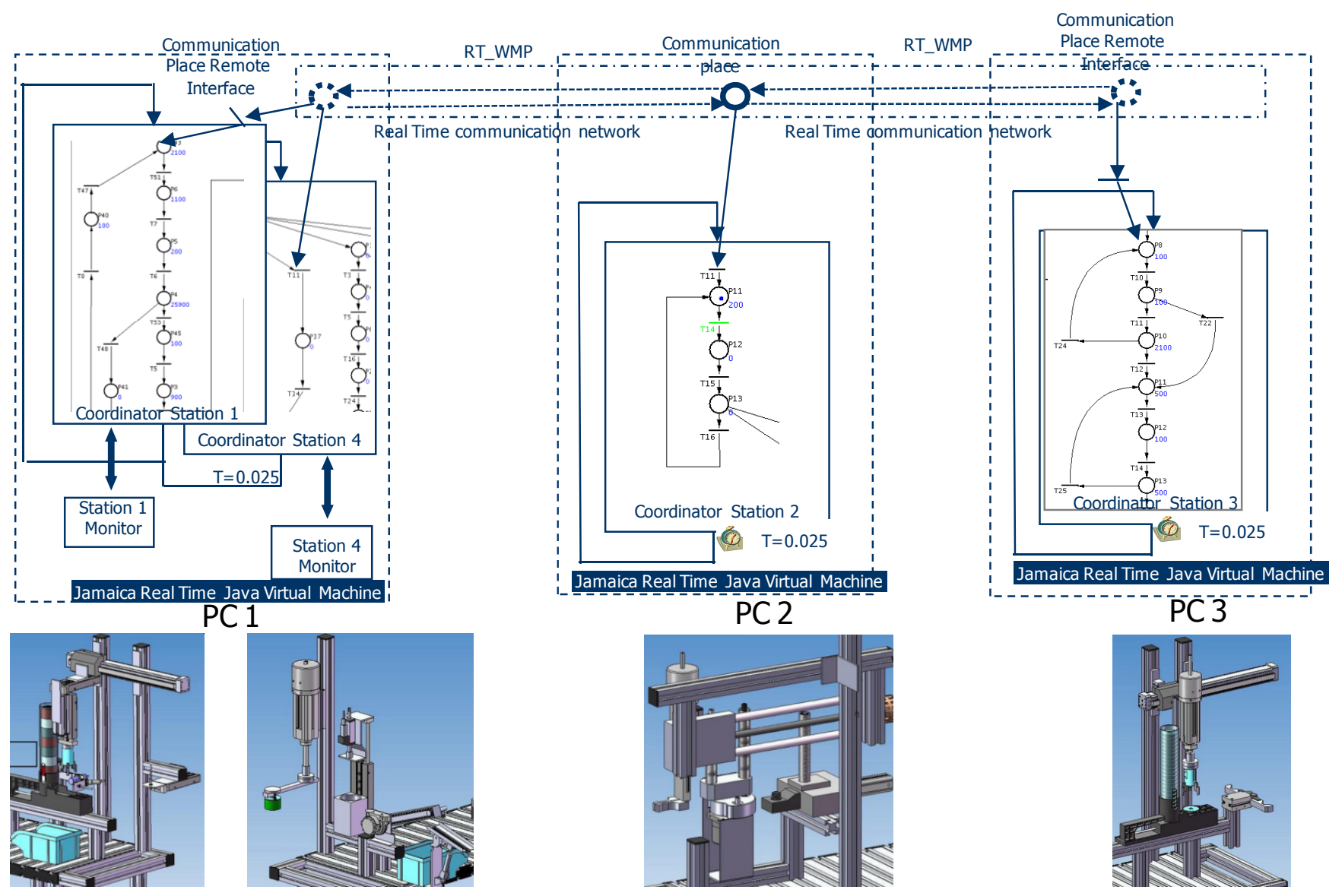

Fig. 7. Software Control Architecture 


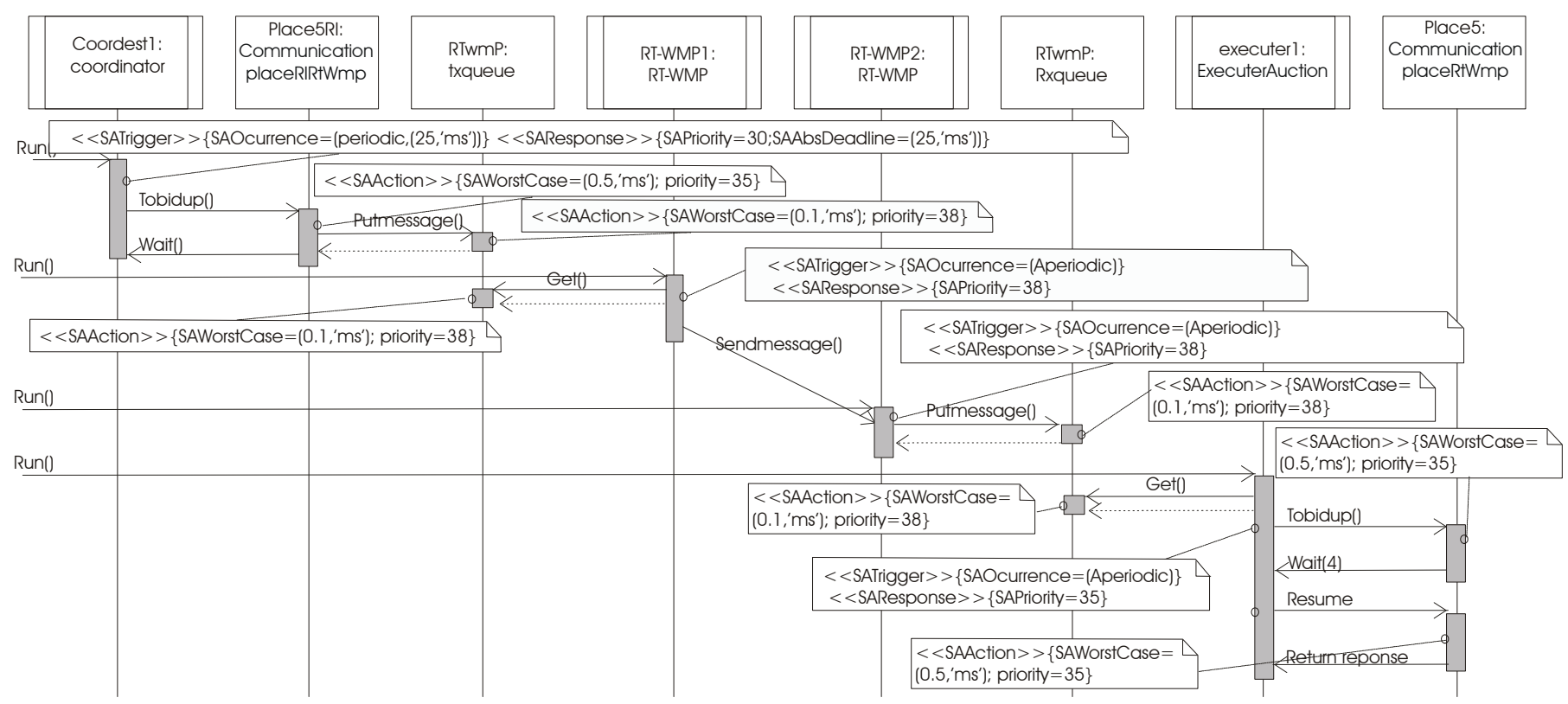

Fig. 8. Communication UML sequence diagram

\section{Control deadlines}

We have also tested the communication time amongst computers and the input/output modules in industrial Ethernet. This time is between 6 and $7 \mathrm{~ms}$. The data transmission time in the Interbus is deterministic and can be known using the characteristics that the manufacturer provides. In this case the data transmission time is $1.6 \mathrm{~ms}$.

With the communication times indicated, a control period of almost $25 \mathrm{~ms}$ is possible. This control period is the period of the coordinators (periodic Real Time Threads).

The proposed concurrent structure and priorities guarantee that the controllers execute forever using updated input data and allow real-time analysis of the thread set. Following a rate monotonic approach, all of the local controller's threads run within their period (the control period) if:

$$
C_{\text {coord }}+t_{\text {bus }}+t_{\text {com }}<=T
$$

Where $T$ is the control period, $C_{\text {coord }}$ is the WCET of the local(s) coordinator(s), $t_{\text {bus }}$ is the WCET for read the inputs and write the outputs in the fieldbus, $t_{\text {com }}$ is the WCET for communicating with a remote communication place.

The WCET $t_{b u s}$ in the fieldbus Ethernet is $7 \mathrm{~ms}$. In the bus Interbus is $1.6 \mathrm{~ms}$. The WCET for communication is $12 \mathrm{~ms}$. In the PC1(fieldbus Interbus):

$$
C_{\text {coordest } 1}+C_{\text {coordest } 4}+t_{\text {bus }}+t_{\text {com }}=1+1+1.6+12=15.6<=25 \mathrm{~ms} .
$$

In the PC2, PC3 and PC4 (fieldbus Ethernet).

$$
\begin{gathered}
C_{\text {coordest } 2}+t_{\text {bus }}+t_{\text {com }}=1.2+7+12=20.2<=25 \mathrm{~ms} .(P C 2) \\
C_{\text {coordest } 3}+t_{\text {bus }}+t_{\text {com }}=1.1+7+12=20.1<=25 \mathrm{~ms} .(P C 3) \\
C_{\text {coordtransport }}+t_{\text {bus }}+t_{\text {com }}=0.9+7+12=19.9<=25 \mathrm{~ms} .(P C 4)
\end{gathered}
$$

If the previous condition is fulfilled, the worst-case response time for events in the system can be calculated as:

$$
t_{r} \leq 2 T+t_{b u s}
$$

That is, the response time $\left(t_{r}\right)$ has a bound related to the control period $(T)$ and the reading-writing time of the bus $\left(t_{b u s}\right)$. An example of system response time to an incoming event is presented in Fig. 9.

In our application, the control period is $25 \mathrm{~ms}$, enough for the dynamics of the controlled system. With Ethernet, the maximum read-write time is $7 \mathrm{~ms}$; therefore, the response time of the real-time control will be:

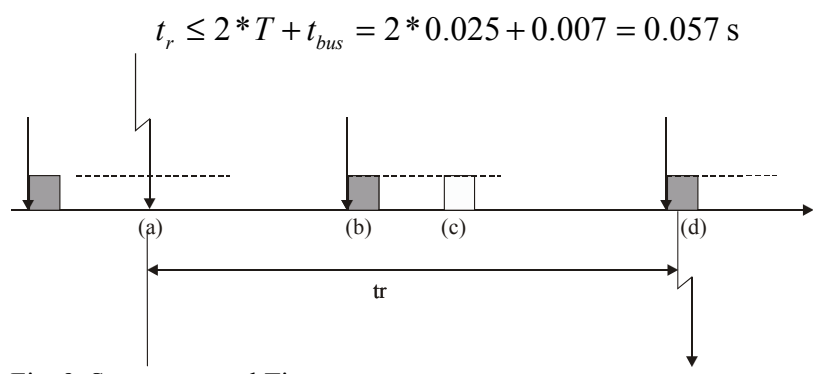

Fig. 9. System control Time response:

a) An event happens in the system (e.g., a pallet arrives at a station).

b) The event is copied to the memory image of the control system.

c) A local controller reads the event and establishes the reaction as changes on memory image of outputs.

d) The outputs are established in the system through the fieldbus.

\section{CONCLUSIONS}

In this paper a new PN implementation technique called distributed coordinators has been developed. This technique involves the use of centralized, decentralized and distributed implementations. 
A platform that allows the implementation of distributed $\mathrm{PN}$, has been developed. For this purpose, a set of Java Classes has been designed to implement synchronous and asynchronous communication paradigms. These classes implement the communications places and its remote interfaces: communications places remote interface, the auction communication place and the Auction solver. We proposed a method to implement simple conflicts in distributed PN. In future works we want to study multiple and coupled conflicts.

To grant the real-time execution of the remote calls to the methods of the remote communication places, a software architecture has been implemented on top of the real-time protocol RT-WMP. In this way the system analyzable from the real time perspective.

All of the techniques and technologies presented in this work have been evaluated in a practical application: the distributed control of a flexible manufacturing cell. The control system of the cell is currently running without problems.

This work, which is an extension of our previous research, provides the basis for further investigations into PN software implementations, languages, and real-time execution platforms.

\section{ACKNOWLEDGMENTS}

This work was funded by the NERO project DPI2006-07928 of the Spanish Ministry of Science and Technology. We want to thanks to José Javier Merseguer for its helpful aid

\section{REFERENCES}

[1] R. Piedrafita and J.L. Villarroel. Petri nets and Java. Real-Time Control of a flexible manufacturing cell. 11th IEEE International Conference on Emerging Technologies and Factory Automation. Prague, September 2006

[2] D. Tardioli, J.L. Villarroel. Real-Time communications over 802.11: RTWMP. The Fourth IEEE International Conference on Mobile Ad-hoc and Sensor Systems. Pisa October 2007
[3] T. Murata. Petri nets: Properties, Analysis and Applications. Proc. of the IEEE, 77(4), 1989, 541-580.

[4] D. Taubner. On the implementation of Petri Nets. Advances in Petri Nets 1988, volume 340 of Lecture Notes in Computer Sciences, pages 418 419, Springer-Verlag, Berlin, Germany, 1988.

[5] G. Bruno, G. Marchetto. Process-translatable Petri Nets for the rapid prototyping of Process Conrol Systems. IEEE Transactions on software Engineering, Vol. SE-12, NO.2. pages 346-357. February 1986.

[6] G.W. Brams, editor. Reseaux de Petri. Theorie et practique. Masson, 1983.

[7] J.L. Briz and J.M. Colom. Implementation of Weighted Place / Transition Nets based on Linear Enabling Functions. Proc of 15th International Conference on Applications and Theory of Petri Nets. pages 99-118. Univ. Zaragoza, 1994

[8] J.M. Colom, M. Silva, and J.L. Villarroel. On software implementation of Petri nets and coloured Petri nets using high-level concurrent languages. In Proc of 7th European Workshop on Application and Theory of Petri Nets, pages 207-241, Oxford, July 1986.

[9] A. Borg, A. Wellings A Real-Time RMI Framework for the RTSJ. Proceedings of the 15th Euromicro Conference on Real Time Systems. Porto, July 2003

[10] J.M. Martínez, M. González Harbour, and J.J. Gutiérrez. "RT-EP: RealTime Ethernet Protocol for Analyzable Distributed Applications on a Minimum Real-Time POSIX Kernel". Proceedings of the 2nd International Workshop on Real-Time LANs in the Internet Age, RTLIA 2003, Porto (Portugal), July 2003.

[11] The Real-Time for Java Expert Group. The Real-time Specification for Java. https://rtsj.dev.java.net/. Addison Wesley, 2000.

[12] R. Piedrafita and J.L. Villarroel. Implementation of Time Petri Nets in Real-time Java. The 4th International Workshop on Java Technologies for Real-time and Embedded Systems - JTRES 2006. Paris, October 2006.

[13] Aicas GmbH. JamaicaVM Realtime Java Technology. http://www.aicas.com/jamaica.html

[14] F.J. García, J.L. Villarroel: "Decentralized Implementation of Real-Time Systems Using Time Petri Nets. Application to Mobile Robot Control". Proc. 5th IFAC Workshop on Algorithms and Architectures for Real-time Control AARTC'98. pp. 11-16, Cancún, México. April 1998.

[15] J.L. Villarroel. Integración Informática del Control de Sistemas Flexibles de Fabricación. PhD thesis, Dpto. de Ingeniería Eléctrica e Informática, Universidad de Zaragoza, Septiembre 1990.

[16] Phoenix Contact. Inline Modular. www.phoenixcontact.com.

[17] Hilscher GmbH. PC cards. http://www.hilscher.com

[18] Schneider Electric. Advantys STB. http://www.telemecanique.com

[19] Pepperl\&fuchs IVI-KHD2-4HRX DataSheet. http://www.pepperlfuchs.com

[20] Bruce Powel Douglass. "Real Time UML: Advances in the UML for Real-Time Systems". Addison Wesley, 2006. 